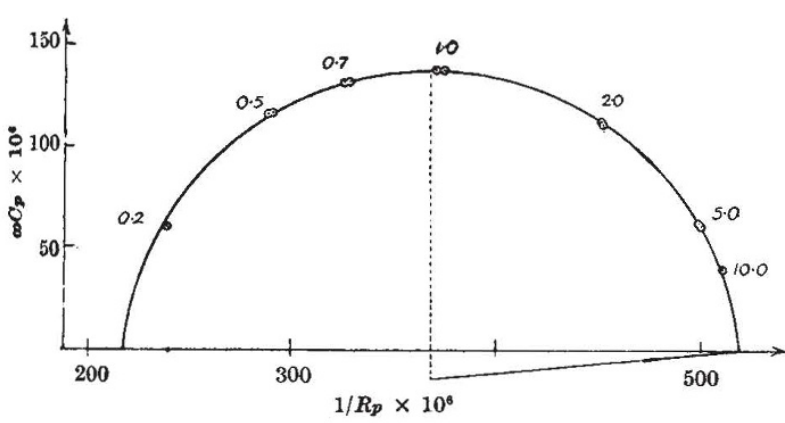

Fig. 2. Time admittance loous of Same rgg as in Fig. 1, SHOWING THE CO-ORDINATES $1 / R_{p}, \omega C_{p}$, OSCILIATING ALONG AN ARC OF A CIRCLE. FREQUENCIES IN KILOCYCLES

that membrane resistance is involved. The membrane phase angle is $84^{\circ}$, and the membrane impedance is of the form $|z|=K \omega^{-2 \varphi / \pi}$. There is no change of phase-angle during the impedance cycle.

The meaning of these impedance cycles will only become clearer when eggs, or other cells which exhibit this phenomenon, are put through their physiological or biochemical paces. So far, these eggs are the only living material in which such changes have been observed; they may be peculiar to this type of egg, which is morphologically somewhat specialized. On the other hand, these eggs are specially suitable for very sensitive measurements of bio-electrical properties, as they present the experimenter with a thin spherical shell of protoplasm the area of which is nearly $1 \mathrm{sq}$. cm. Other material might not be so favourable for the observation of these rhythmical changes in protoplasmic structure.

Zoological Laboratory, ROTHSCHILD

Downing Street,

Cambridge.

Dec. 10.

${ }^{1}$ Hubbard, M. J., and Rothschild, Lord, Proc. Roy. Soc., B, 127, 510 (1939).

${ }^{2}$ Rothschild, Lord, Nature, 145, $7 \pm 4$ (1940).

'Rothschild, Lord, J. Exp. Biol., in the press.

${ }^{4}$ Cole, K. S., and Curtis, H. J., J. Gen. Physiol., 22, 37 (1938).

\section{Unco-ordinated Growth in Paramecium Induced by 'Gammexane'}

THE $\gamma$-isomer of hexachlorocyclohexane ('Gammexane') is toxic to Paramecium caudatum when introduced into the cultures at a rate of 10 parts per million down to 1 per million. The action of the poison is slow and cumulative, the organisms continuing to feed until near death. Nuclear division is inhibited, and so they become hypertrophied, increasing in length and breadth up to 50 per cent beyond normal. There is a threshold concentration of the drug somewhere between 1 and 0.5 parts per million where some normal division occurs, but a proportion of the hypertrophied cells divide as to the nuclei but not as to the body of the cell itself. Consequently, Siamese-twin forms are produced which feed and live a long time without making further progress, except that a small lateral outgrowth sometimes suggests that they might produce buds. Complete budding has not been observed in the 'Gammexane'-treated medium.

When the twin forms, or highly hypertrophied Paramecium from the lower 'Gammexane' concentra. tions, are placed in normal medium, macronuclei and micronuclei divide repeatedly while the cell throws out lobes in all directions. As many as fourteen micronuclei have been counted in one individual while the macronucleus was throwing off pieces of varying size. Buds become detached with varying nuclear combinations, and while it is likely that all these buds are not viable, some of them succeed in starting fresh colonies of apparently normal individuals. These budding forms have several times the bulk of a normal Paramecium, are ciliated all over in the normal way, and have a pharynx or sometimes two pharyngeal openings. They feed, and the cilia are active; small ones swim, but the large ones contort themselves and roll over constantly but are too heavy to move from the floor. The accompanying illustration shows the normal form of Paramecium and some of the smaller abnormal forms drawn from life. It should be noted that while the pressure of the drug is on them, only simple Siamese twins

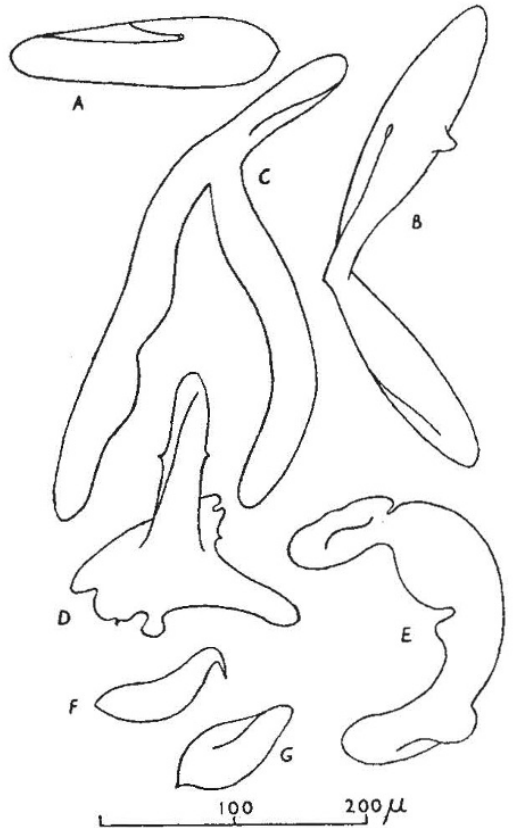

NORMal Paramecium aNd Forms DISTORTED BY 'GAMMEXaNE'. (a) NORMAL; (b) THF SIAMESE TWINS; $(c),(d),(e)$ SMALI BUDDING FORMS; $(f),(g)$ FREED BUDS. ALL ARE FULLY CILIATED.

are formed; but when this pressure is relieved the fully unco-ordinated growth occurs. In a manuscript on this topic by the late J. C. Mottram, who has recorded these abnormal growths in Paramecium after contact with several of the cyclic hydrocarbons ${ }^{1}$, emphasis is also laid on the two factors, one sensitizing and the other developing.

This development arises out of my connexion with the informal committee on sewage filter-flies of the Water Pollution Research Board (Department of Scientific and Industrial Research). My thanks are due to the officers of the Board for the supply of materials. A full account of the method of obtaining these abnormalities and of the behaviour so far observed will shortly be ready for publication.

University, Lx. LLOY D

Leeds.

Nov. 30 .

'Mottram, J. C., “The Problem of Tumours" (London, 1942). 